\title{
Use and uncertainties of mutual information for computed tomography/ magnetic resonance (CT/MR) registration post permanent implant of the prostate
}

\author{
Peter L. Roberson ${ }^{\text {a) }}$ \\ University of Michigan, Department of Radiation Oncology, Ann Arbor, Michigan 48109 \\ P. William McLaughlin and Vrinda Narayana \\ University of Michigan, Department of Radiation Oncology, Ann Arbor, Michigan 48109 \\ and Providence Cancer Center, Department of Radiation Oncology, Southfield, Michigan 48075
}

Sara Troyer

University of Michigan, Department of Radiation Oncology, Ann Arbor, Michigan 48109

George V. Hixson

Providence Cancer Center, Department of Radiation Oncology, Southfield, Michigan 48075

Marc L. Kessler

University of Michigan, Department of Radiation Oncology, Ann Arbor, Michigan 48109

(Received 14 May 2004; revised 18 October 2004; accepted for publication 26 November 2004; published 26 January 2005)

\begin{abstract}
Post-implant dosimetric analysis for permanent implant of the prostate benefits from the use of a computed tomography (CT) dataset for optimal identification of the radioactive source (seed) positions and a magnetic resonance (MR) dataset for optimal description of the target and normal tissue volumes. The CT/MR registration process should be fast and sufficiently accurate to yield a reliable dosimetric analysis. Since critical normal tissues typically reside in dose gradient regions, small shifts in the dose distribution could impact the prediction of complication or complication severity. Standard procedures include the use of the seed distribution as fiducial markers (seed match), a time consuming process that relies on the proper identification of signals due to the same seed on both datasets. Mutual information (MI) is more efficient because it uses image data requiring minimal preparation effort. A comparison of MI registration and seed-match registration was performed for twelve patients. MI was applied to a volume limited to the prostate and surrounding structures, excluding most of the pelvic bone structures (margins around the prostate gland were $\sim 2 \mathrm{~cm}$ right-left, $\sim 1 \mathrm{~cm}$ anterior-posterior, and $\sim 2 \mathrm{~cm}$ superior-inferior). Seeds were identified on a $2 \mathrm{~mm}$ slice CT dataset using an automatic seed identification procedure on reconstructed three-dimensional data. Seed positions on the $3 \mathrm{~mm}$ slice thickness T2 MR data set were identified using a point-and-click method on each image. Seed images were identified on more than one MR slice, and the results used to determine average seed coordinates for MR images and matched seed pairs between CT and MR images. On average, 42\% (19\%-64\%) of the seeds (19-54 seeds) were identified and matched to their CT counterparts. A least-squares method applied to the CT and MR seed coordinates was used to produce the optimum seed-match registration. MI registration and seed match registration angle differences averaged 0.5 degrees, which was not significantly different from zero. Translation differences averaged 0.6 (1.2 standard deviation) mm right-left, $-0.5(1.5) \mathrm{mm}$ posterior-anterior, and $-1.2(2.0) \mathrm{mm}$ inferior-superior. Registration error estimates were approximately $2 \mathrm{~mm}$ for both the MI and seed-match methods. The observed standard deviations in the offset values were consistent with propagation of error. Registration methods as applied here using mutual information and seed matching are consistent, except for a small systematic difference in the inferior-superior axis for a minority of cases $(\sim 15 \%)$. Cases registered with mutual information and with bony anatomy misregistration of greater than $\sim 5 \mathrm{~mm}$ should be evaluated for rescan or seed-match registration. The improvement in efficiency of use for the MI registration method is substantial, $\sim 30 \mathrm{~min}$ compared to several hours using seed match registration. (C) 2005 American Association of Physicists in Medicine. [DOI: 10.1118/1.1851920]
\end{abstract}

Key words: mutual information, prostate brachytherapy, registration, MR, CT

\section{INTRODUCTION}

The American Brachytherapy Society guidelines for post implant dosimetry specify the use of computed tomography
(CT) imaging. ${ }^{1}$ While $\mathrm{CT}$ imaging is very sensitive to radioactive source (seed) locations, it provides a relatively poor definition of prostate (target) and sensitive (nontarget) tissue volumes compared to magnetic resonance (MR) imaging., ${ }^{2,3}$ 
Anatomic distortion due to edema and reduction in tissue edge contrast on CT due to the presence of the seeds aggravate this problem.

No single imaging modality offers optimal visualization of seed positions, target and normal tissue structures. CT remains the optimal choice for the identification of seed positions. MR T2 scans can best provide visualization of anatomical structures. ${ }^{3,4}$ While other MR imaging sequences can better visualize seed positions compared with $\mathrm{T} 2,{ }^{5,6}$ seed identification by MR remains less than acceptable for dose calculation to normal structures surrounding the prostate gland. Registration of MR T2 and CT data sets offers the optimal description of seed position and anatomy needed for the post implant evaluation.

Previous methods used for registration have included anatomic surface and feature matching ${ }^{7}$ and seed matching techniques, or a combination. Surface matching techniques suffer from the inconsistency of volume definitions inherent in the sensitivity of the imaging modalities. Seed matching techniques require the identification of images of the same seeds on the CT and MR data sets. This is a time-intensive process prone to problems with like-seed identification error.

Alternatively, post-implant dosimetry may be accomplished with the MR scan alone. ${ }^{5}$ The CT scan may be used for guidance during source localization on the MR scan. MR sequences that are a compromise specific to seed identification and volume definition may be chosen. Alternatively, multiple scans may be performed as part of the same study to improve the optimum success of seed identification and volume identification. This process is technically demanding requiring more highly trained staff, and can be prone to misinterpretation. ${ }^{5}$

The mutual information (MI) technique is an automated approach for the registration of large data sets. ${ }^{8,9}$ As used here, the procedure requires two three-dimensional (3D) image data sets of the same anatomic volume. The result is a rigid rotation-translation of a data set to be registered to the reference data set. The voxels in each image data set are considered random samplings of a random variable. A probability density function of two random variables is defined to represent the corresponding voxel values in the two data sets. If two image data sets are approximately registered, the voxels are less jointly random. Voxels with clustered intensity values on one image data set will correspond to voxels with clustered intensity values on the other data set. Thus, there may be multiple clusters of voxels with correlated intensities from the two data sets. The joint probability density function becomes more clustered as the two data sets become better registered. Optimal registration is achieved by maximizing the probability density function estimating the mutual information contained in the two data sets.

Because of the difference in scan environment between $\mathrm{CT}$ and MR (i.e., table and presence of the pelvic coil) and the time interval between scans, a potentially significant displacement of the prostate gland relative to the bony anatomy is possible. The registration results depend on the quantity of information used for the registration. To achieve the optimal registration of the prostate and neighboring tissues, it was necessary to study the influence of limiting the volume of interest (VOI) used for registration. While selectivity in local registration is inherently applied during the surface or seed match registration processes, it must be explicitly chosen (e.g., VOI and initial conditions) and can be better controlled using MI registration.

Each registration technique has a unique metric used to determine optimum registration. Ultimately, the user must decide to accept or reject a registration based on perceived similarities of anatomical structures. This final decision represents yet another metric applied in a pass-fail mode. While all metrics are limited by the resolution of the data sets, other influences may yield a systematic discrepancy between metrics. The comparison of registration techniques will result in a difference potentially greater than the uncertainty of either technique as defined by its metric. The intent of this paper is to quantify the difference between current and past registration techniques (MI vs seed match), while assessing the validity of the MI technique in the context of post-permanent implant of the prostate.

\section{MATERIALS AND METHODS}

\section{A. Scanning and seed definition}

Twelve patients were chosen for the comparison of MI registration to a rigorous seed match registration. Six patients received primary brachytherapy treatment prescribed to 145 Gy and six received a brachytherapy boost prescribed to 108 Gy in conjunction with 45 Gy external beam treatment. The average number of seeds implanted was 74 per case (range 43-112). Two weeks following the implant, an axial CT image data set (2 $\mathrm{mm}$ slice thickness, no gap) and an axial MR T2 image data set $(\mathrm{TE}=96 \mathrm{~ms}, \mathrm{TR}=4500 \mathrm{~ms}$, $3 \mathrm{~mm}$ slice thickness, no gap) were obtained. The MR data set was collected with a pelvic coil. Sagittal $(5 \mathrm{~mm}$ slice thickness) and coronal $(5 \mathrm{~mm})$ MR images were also obtained. The MR imaging studies were taken within two hours of the CT imaging study to minimize anatomic differences. Prostate contours were entered on all data sets. Registration of coronal and sagittal MR data with axial MR data allowed the refinement of the prostate volume definition. After mutual registration of MR data sets, the axial MR data set was registered to the CT data set. Sagittal and coronal data sets were moved with the axial MR data set, completing the MR to $\mathrm{CT}$ registration process.

Seed positions were identified from the CT data set using the University of Michigan treatment planning system (UMPLAN) auto-recognition software. Orthogonal views of the region-of-interest digitally reconstructed radiographs were created from the CT data set for visual review of seed localization. The voxel space used for localization was defined by the minimum pixel size in the original images, approximately $1 \times 1 \times 2 \mathrm{~mm}^{3}$. Cubic voxels were defined by linear interpolation between slice planes. An intensity map was determined using the convolution of a Gaussian kernel with $0.5 \mathrm{~mm}$ sigma with the voxel space. A peak finder scanned the intensity map to yield local maxima, which were defined as potential seed locations. Three plane films (AP and $+10^{\circ}$ 
caudad and $10^{\circ}$ cephalad) taken at the time of the CT study were used to help identify artifacts (rare) or seeds too close to be resolved (doublets). Typical initial localization successfully identified nearly all seed locations correctly. Seed doublets or artifacts were easily identified via the plane films and appropriate corrections to the seed distribution were performed.

\section{B. Seed match registration}

Potential seed positions were identified on the axial MR T2 data sets on all axial slices. Seed positions were sorted and coordinates were averaged for the same seeds identified on more than one axial scan. The seed distribution was compared to that identified by CT using an approximate (anatomic surface) $\mathrm{MR} / \mathrm{CT}$ registration. A translational coordinate search was used to optimize the number of matched pairs using a maximum match distance criterion of 3 or $4 \mathrm{~mm}$. The seed matches were checked visually on the axial scans to ensure a proper match and to eliminate seeds too close to their neighbors to provide positive identifications. On average, $42 \%$ (ranges of 19\%-64\% and 17-54 seeds) of the implanted seeds were matched. Using the matched set of coordinates, the seed match registration was determined by a least-squares (LS) fit. The result was a $4 \times 4$ transformation matrix describing the rigid body rotation and translation required for optimal registration as defined by the matched seed distributions.

\section{Mutual information registration}

Software routines for MI registration were implemented compatible with the treatment planning system designed at the University of Michigan (UMPLAN) ${ }^{10}$ used to perform the post implant dose calculations. The MI routine used a Nelder-Mead simplex algorithm to iteratively perturb the higher resolution dataset. Each new candidate transformation was used to reformat the higher resolution data set at the voxel locations of the lower resolution data set. Reformatting the higher resolution data set minimized the impact of the reformatting process on the MI calculations. The mutual information between the datasets was computed and compared to the previous iteration. Iterations continued until the mutual information was maximized and the relative change between successive iterations fell below a threshold value. The MI technique is sensitive to maximum overlap between regions of relatively uniform image densities present in both datasets (e.g., bone, muscle, fat). Input parameters are (1) the volume proposed for registration (cropped volume of interest for the lower resolution dataset), (2) the control point positions determined by operator identified landmarks (minimum of 3 points required), and (3) the search range of the algorithm. The MI registration process was performed efficiently $(<30 \mathrm{~min})$ and visually inspected for validity. Visual inspection tools included transparent overlay and sliding bar or wiper comparison between scan planes.

The MI technique defined one of the data sets as the reference while the other was adjusted to maximize the mutual information. Only rigid adjustments were allowed (rotations and translations). Because of the freedom for rotations, the data set being adjusted did not necessarily remain with its major axes aligned to the reference data set. Reconstructed planes from the adjusted data set were compared to and judged against original planes in the reference data set. Best advantage of the information contained in the data sets suggested that the data set with the higher resolution (e.g., CT) be adjusted and compared to the lower resolution set (e.g., MR). The MI software exported to UMPLAN the optimal transformation matrix for the movement of the lower resolution dataset (e.g., MR).

The registration process resulted in a $4 \times 4$ transformation matrix describing rigid rotation and translation. The MR data sets were mutually registered and then subsequently registered to the post-implant CT data set. The MR axial data set was aligned to the MR sagittal dataset through MI to yield a transformation matrix for the sagittal dataset $T(\mathrm{Ms} \rightarrow \mathrm{Ma})$. The MR axial dataset was aligned to the MR coronal data set through MI to yield a transformation matrix $T(\mathrm{Mc} \rightarrow \mathrm{Ma})$. The CT axial data set was aligned to the MR axial data set to yield the transformation matrix $T(\mathrm{Ma} \rightarrow \mathrm{CT})$. The net rotation-translation matrix for the sagittal dataset was obtained by matrix multiplication of the sagittal MR transformation matrix with the axial MR to axial CT matrix

$$
T(\mathrm{Ms} \rightarrow \mathrm{CT})=T(\mathrm{Ma} \rightarrow \mathrm{CT}) \cdot T(\mathrm{Ms} \rightarrow \mathrm{Ma}),
$$

where the dot represents $4 \times 4$ matrix multiplication. The coronal data set registration matrix was similarly calculated.

During the MR-axial-to-CT-axial registration process, VOI's were determined by cropping the MR axial data set. This was necessary to avoid registration being influenced by structures beyond the local environment. The internal structures of the MR and CT data sets were influenced by the patient setup and changes in bladder and rectum filling. The registration volume was selected by cropping down to the desired rectilinear box. A default cropping was used to limit the influence of the surrounding structures, while still retaining the local information immediately adjacent to the prostate. Additional tests were performed using more severe cropping with specific goals. However, when all additional cropping was used simultaneously, insufficient volume remained to avoid sufficient rotational symmetry to confuse the MI algorithm, as evidenced by large rotation angles. Cropping definitions used are listed below.

Default VOI. The image was cropped laterally just inside of the pelvic bones at mid-prostate; anteriorly to include approximately half of the pubic bone; and posteriorly at mid rectum. The images were not cropped superiorly or inferiorly, since the scan intervals on the MR data sets were sufficiently tight ( $\sim 2 \mathrm{~cm}$ margin on the prostate gland).

Rectum Crop. The posterior border of the default VOI was adjusted to substantially remove the rectum.

Bladder Crop. The superior margin of the default VOI was decreased.

Bone Crop. The anterior, lateral, and inferior margins of the default VOI were reduced, effectively removing the remaining bone. 
The MI process depends on the choice of starting position and volume of interest. For the highly cropped volumes, a reasonably close starting position (within $\sim 5 \mathrm{~mm}$ and $\sim 5$ degrees) must be chosen to allow the optimization process to yield a valid registration. Since bladder filling or other anatomical changes were possible, bony landmarks were not always reliable. Matching points were chosen from the soft tissue in and surrounding the prostate gland. Seed positions were also used in some cases. For axial-to-axial registration, it was typically sufficient to choose three points on matched axial images, although reconstructed sagittal planes were used for cases with pelvic rotation differences. The sagittal views used for the cropping were reconstructed from the MR axial data set. Volumes of interest generated from expansions of the prostate gland were less effective because of the increased difficulty of including soft tissue while excluding bone.

The sagittal or coronal data set registrations used the full datasets and were relatively insensitive to the starting position and search range.

\section{Registration comparison}

Registration comparisons were performed by calculating angle and translation differences in the three orthogonal dimensions. Angle differences were expressed as axis rotation angles derived from the directional cosines for each axis (the diagonal elements $T_{x x}, T_{y y}$, and $T_{z z}$ in the $3 \times 3$ rotation submatrix). For rotation along the $x$ axis (rotation in the $y-z$ plane)

$$
\left.\operatorname{Cos} \theta_{x}=\sqrt{\left(T_{y y}\right.} T_{z z} / T_{x x}\right) .
$$

Corresponding formulas were used for the other axes. Net rotation differences were expressed as simple differences between axis rotation angles.

Translation differences depended on location if there was also a rotation difference. An unbiased estimate of the translation difference was determined by calculating the translation of the center-of-mass of the seed distribution. Given a seed distribution identified on CT, the coordinates of the center-of-mass of the distribution were the mean coordinates of the individual seed coordinates (vector $R_{\mathrm{CM}}$ ). Each registration run produced a $4 \times 4$ rotation-translation matrix, $T_{i}$, representing the needed change in the axial MR dataset for registration experiment ' $i$ ' to match the CT dataset. Then, $T_{i} \cdot R_{\mathrm{CM}}^{i}=R_{\mathrm{CM}}$ represents the mapping of the vector for the position in the MR data set that mapped to the seed distribution center of mass in the CT coordinate system. Inverting this equation, $R_{\mathrm{CM}}^{i}=T_{i}^{-1} \cdot R_{\mathrm{CM}}$. Differences in registration were defined as coordinate differences between center-ofmass vectors $\left(R_{\mathrm{CM}}^{i}\right)$.

The CT seed center-of-mass position was translated to the axial MR coordinate system by applying the transformation matrix generated from the MI or the seed match with LS registration processes:

$$
R_{\mathrm{CM}}^{\mathrm{MI}}=T_{\mathrm{MI}}^{-1} \cdot R_{\mathrm{CM}}
$$

and

$$
R_{\mathrm{CM}}^{\mathrm{LS}}=T_{\mathrm{LS}}^{-1} \cdot R_{\mathrm{CM}},
$$

where $T$ represents the $4 \times 4$ registration matrix and $R$ represents the $4 \times 1$ translational vector, consisting of $1 \times 3$ spatial coordinate transformation and a 1 . The translational difference was calculated as the difference in each of the first three elements of $R_{\mathrm{CM}}$

\section{E. Registration uncertainty}

There is a registration uncertainty associated with each registration technique. This uncertainty is a function of the metric used by the registration technique and the quality of the input data.

\section{Seed match with least squares}

Seed-match registration accuracy is no better than the user's ability to identify seed coordinates and to match images for the same seed in each data set. Assuming that the seed images are correctly matched, the uncertainty of the registration is dependent on the uncertainty of the identified coordinates. In the axial plane $(x-y)$, the position uncertainty is limited by the pixel resolution $(\sim 1 \mathrm{~mm}$ for both $\mathrm{CT}$ and MR data sets) and the coordinate input error. The $x-y$ uncertainty is primarily random, implying a smaller registration error than average seed coordinate error. Thus the net estimated $x-y$ uncertainty of registration is less than $1 \mathrm{~mm}$. In the superior-inferior $(z)$ axis, the uncertainty is dependent on the slice resolution and could have a significant systematic component. Since the seed positions on CT were determined by a seed search algorithm, and the CT slice thickness $(2 \mathrm{~mm})$ is less than the MR slice thickness $(3 \mathrm{~mm})$, the dominant $z$ uncertainty is expected to be associated with the MR seed position identification process. To minimize the $z$ uncertainty, the seed signals on MR were tracked on multiple axial slices. The signal level (holes) varied with intensity depending on the relative position of the slice and the seed position and orientation. Nearly all seeds were a part of a string of seeds, and thus were positioned close to parallel with the $z$ axis ( within 30 degrees).

The slice-plane contribution to the registration error was studied by forming an ideal seed distribution and mathematically slicing the image planes in 2, 3, and $5 \mathrm{~mm}$ thicknesses. The seed distribution contained 50 seed positions in 10 strings, each with $1 \mathrm{~cm}$ seed spacing. Each sliced seed or seed fragment was collapsed to the slice center. Seed images with less than $1 / 3$ of the seed volume were discarded (discriminator effect). The remaining seed positions were moved within the plane of the slice using a random $1 \mathrm{~mm}$ Gaussian blurring function and quantized to the nearest pixel position. Seed positions were matched from multiple slices to form the final seed coordinates. Seed coordinate deviations from the original (ideal) positions were compiled.

\section{Mutual information}

The MI dataset registration process depended on the assumption that the data sets represent the same anatomical structures. The goal was to overlay the anatomical informa- 
TABLE I. Average and root-mean square displacement of matched seeds used for seed match registration.

\begin{tabular}{|c|c|c|c|c|c|c|c|c|c|c|c|c|c|}
\hline Case & $\mathrm{P} 1$ & $\mathrm{P} 2$ & P3 & $\mathrm{P} 4$ & P5 & P6 & P7 & P8 & P9 & $\mathrm{P} 10$ & $\mathrm{P} 11$ & $\mathrm{P} 12$ & Average \\
\hline$x,{ }^{\mathrm{a}} \mathrm{mm}$ & 0.7 & 0.8 & 0.8 & 0.8 & 0.7 & 1.1 & 0.6 & 1.3 & 1.3 & 0.6 & 0.6 & 0.8 & 0.8 \\
\hline$y,{ }^{\mathrm{a}} \mathrm{mm}$ & 0.7 & 1.0 & 0.9 & 0.7 & 0.7 & 1.4 & 0.8 & 1.4 & 1.1 & 0.8 & 0.9 & 1.2 & 1.0 \\
\hline$z,{ }^{\mathrm{a}} \mathrm{mm}$ & 1.3 & 1.4 & 1.5 & 1.6 & 1.4 & 1.6 & 1.2 & 1.8 & 1.7 & 1.3 & 1.7 & 1.6 & 1.5 \\
\hline $\mathrm{RMS}^{\mathrm{b}}{ }^{\mathrm{mm}}$ & 2.1 & 2.6 & 2.2 & 2.3 & 2.1 & 2.8 & 1.9 & 3.1 & 2.9 & 2.2 & 2.4 & 2.6 & 2.4 \\
\hline Seed Pairs & 47 & 32 & 17 & 19 & 54 & 23 & 52 & 30 & 19 & 30 & 21 & 24 & 31 \\
\hline
\end{tabular}

${ }^{\mathrm{a}}$ Average absolute displacement in orthogonal dimensions. $x$ : Left-right. $y$ : Posterior-anterior. $z$ : Inferior-superior (perpendicular to CT image plane).

${ }^{\mathrm{b}} \mathrm{RMS}$ : root mean square.

tion to achieve a perfect match of the images of the physical body from which both data sets were derived. However, the separation of scans in time and the dissimilar patient setup in the scanners limited the precision of registration. Possible causes for anatomic variations are (1) the presence of the MR pelvic coil, and (2) changes in anatomical relationships between organs (e.g., changes in the filling of the bladder and/or rectum).

MI registrations were performed repeatedly while varying the input parameters to determine the uncertainty of the maximum in the mutual information metric. The MR axial to $\mathrm{CT}$ axial registration process varied the initial starting positions only (using standard crop), while the MR coronal or sagittal to MR axial registrations varied the initial starting point and search range.

\section{RESULTS}

Seed match registration was compared to MI registration for 12 test cases. Registration differences are quoted in terms of relative rotation angle and translation offset for the three orthogonal axes ( $x$ : left to right, $y$ : posterior to anterior, and $z$ : inferior to superior). Mean differences with standard errors and root-mean square (RMS) differences representing total offset at the center-of-mass of the seed distribution are presented.

\section{A. Seed match registration}

The LS calculation minimized the displacement between matched seed locations. Average displacements and average RMS displacements are given in Table I. Also listed are the total numbers of identified seed pairs used for the registration. The global average RMS displacement was $2.4 \mathrm{~mm}$ for the 12 test cases. The average absolute displacement was greatest in the $z$ dimension $(1.5 \mathrm{~mm})$, attributed to the greater uncertainty due to the slice thicknesses of the image data sets. Several cases had potential outlier source pairs with approximately $4 \mathrm{~mm}$ of separation. With these pairs removed from the least-squares fit, the net registration was shifted by at most $0.2 \mathrm{~mm}$.

The mathematical slice-plane study resulted in average mean-square seed displacements between image sets of approximately $0.5 \mathrm{~mm}$ in the plane of the slice (approximately $\frac{1}{2}$ of the pixel size). Perpendicular to the plane of the slice, mean-square displacements were $1.1,1.2$, and $1.7 \mathrm{~mm}$ for 2 , 3 , and $5 \mathrm{~mm}$ slices, respectively. For the mathematical study, the residual registration difference from ideal was less than $0.1 \mathrm{~mm}$. The displacements of the seed positions on the slice planes relative to known starting seed images for the three slice thicknesses studied are illustrated in Fig. 1. The larger the slice thickness, the more likely that the central point of the slice will occur far from the center of the seed image. Given the results of the slice plane study, we attribute about half of the observed mean square displacements to the mathematical problem of reconstructing sliced data.

To test the ability of the observer to identify seed images on MR, seeds were followed from slice to slice for a few full strings. While the seed image intensity increased and decreased along the string, it did not totally disappear between seed positions. For the slice thickness of $3 \mathrm{~mm}$, the intensity of each seed appeared to meld into the intensity of the following seed in the string. This is most likely due to the $\sim 5 \mathrm{~mm}$ gap between the seeds on a string and the nonideal profile for the nominal $3 \mathrm{~mm}$ MR slice thickness. MR slice profiles can extend to twice the nominal width with only $\sim 80 \%$ of the signal coming from within the targeted slice. ${ }^{11}$ The identification of seed position is dependent on choosing the most intense seed images. Some seed images chosen on adjacent slices may have been due to different seeds on the same string. This may have confused the seed identification

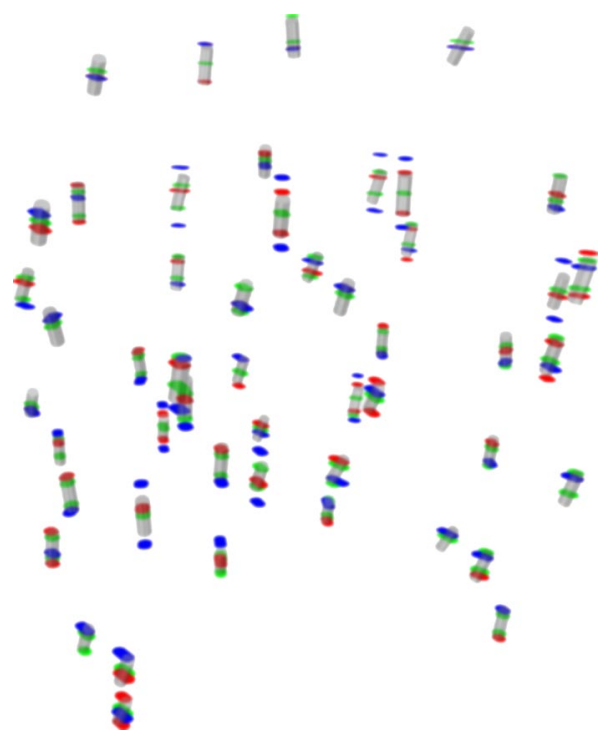

FIG. 1. Slice plane study. Shown are seed volumes and seed image positions if the seed distribution is sliced in $2 \mathrm{~mm}, 3 \mathrm{~mm}$, or $5 \mathrm{~mm}$ increments. 
TABLE II. Mutual information registration uncertainty due to initial parameters. Values are standard deviations of repeat sample registration results. The MR-axial-to-CT-axial registrations used a cropped volume of interest. The MR-coronal- or MR-sagittal-to-MR-axial registrations used the full data sets.

\begin{tabular}{cccccc}
\hline \hline & \multicolumn{3}{c}{ Displacement $^{\mathrm{a}}$} \\
\cline { 2 - 3 } & \multicolumn{3}{c}{$\begin{array}{c}\text { MR Axial to CT Axial } \\
\text { 8 registrations }\end{array}$} & & \multicolumn{3}{c}{$\begin{array}{c}\text { MR Coronal or Sagittal to MR Axial } \\
15 \text { registrations }\end{array}$} \\
\cline { 2 - 3 } \cline { 5 - 6 } Axis & Angle, Deg & Offset, mm & Angle, Deg & Offset, mm \\
\hline$x$ & 0.6 & 0.9 & & 0.2 & 0.1 \\
$y$ & 0.6 & 0.7 & & 0.1 & 0.2 \\
$z$ & 1.1 & 0.3 & & 0.04 & 0.4 \\
RMS $^{\text {b }}$ & 1.3 & 1.2 & 0.3 & 0.4 \\
\hline
\end{tabular}

${ }^{\mathrm{a} A v e r a g e}$ angle or offset in orthogonal dimensions. $x$ : left-right. $y$ : posterior-anterior. $z$ : inferior-superior (perpendicular to CT image plane).

${ }^{b}$ RMS: Root mean square.

algorithm as it searched for similar coordinates on multiple slices, increasing the potential for error along the $z$ axis. Thus, there is a significant contributor to the seed identification error due to observer selection of the seed image on MR, which may have a systematic component.

The net registration error may be less than the average RMS displacements because the registration is determined from a large set of matched seed pairs, greatly decreasing the random contribution to the registration error. Primary contributors to systematic error are the source position determination on MR related to the imperfect imaging provided by $\mathrm{MR}$ and the potentially nonrigid relationship of the seed distribution from CT to MR. The estimated systematic error along the $z$ axis is greater than $1 \mathrm{~mm}$. The total registration error for the seed-match technique was estimated to be $\sim 2 \mathrm{~mm}$.

\section{B. Mutual information}

Table II contains the standard deviations in the angle and offset observed for the MR axial to CT axial registrations (sample of 8) and the MR coronal or sagittal to MR axial registrations (sample of 15). The latter was less dependent on the accuracy of starting position to achieve an acceptable registration. The MR to CT registration using the VOI had less angular sensitivity (RMS angle uncertainty of 1.3 degrees compared to 0.3 degrees) and scan-plane offset sensitivity (RMS offset of $1.2 \mathrm{~mm}$ compared to $0.4 \mathrm{~mm}$ ) compared to the MR to MR registrations. Since the registration tests could not be considered thorough, the random error

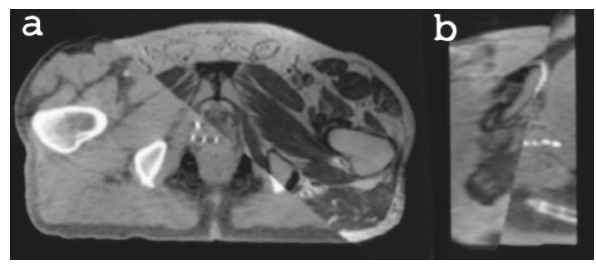

FIG. 2. Axial MR to CT registration verification panel for (a) axial and (b) sagittal views of the wiper comparison tool slicing through mid implant volume. for the MI axial registration process is greater than the 1.3 degrees and $1.2 \mathrm{~mm}$ values quoted. Uncertainties in the range 1.5-2 degrees and 1.5-2 $\mathrm{mm}$ was estimated based on the voxel sizes used to obtain the data sets. An example axial $\mathrm{MR}$ to CT registration verification panel is shown in Fig. 2.

\section{Comparison of registration techniques}

Axial MR to axial CT MI registration results were compared to seed match registration results in 12 cases. Figure 3 shows the net differences in angle and offset in the three dimensions. Translation offsets were greater for the $z$ axis (Inf-Sup) with a negative bias. The average and standard deviation rotation and translation offsets are shown in Table III (default VOI, columns 2-5).

Error for the comparison between registration techniques includes uncertainty from both techniques. Since the metric used for each of the registration techniques was unique, random uncertainties present in the comparison were expected to be greater than either registration process alone. The random error in the comparison registration process was estimated to be on the order of $\sim 2$ degrees and $\sim 2 \mathrm{~mm}$ based on the results given in Tables I and II. A difference in excess of approximately 3 degrees and $3 \mathrm{~mm}$ or $4 \mathrm{~mm}$ RMS was considered outside of random uncertainty and indicative of the presence of potential detectable systematic error. Rota-

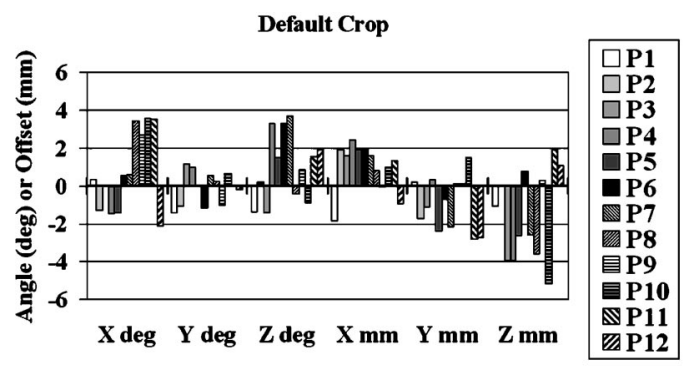

FIG. 3. Mutual information vs seed match registration offsets for default crop. Shown are rotation and translation offsets for twelve cases in the left-right $(x)$, posterior-anterior $(y)$, and inferior-superior $(z)$ directions. 
TABLE III. Average differences in registration comparing mutual information relative to seed match registration for 12 test cases.

\begin{tabular}{|c|c|c|c|c|c|c|c|c|}
\hline \multirow[b]{3}{*}{ Axis } & \multicolumn{4}{|c|}{ Default $\mathrm{VOI}^{\mathrm{a}}$} & \multicolumn{4}{|c|}{ Optimum VOI } \\
\hline & \multicolumn{2}{|c|}{ Angle, deg } & \multicolumn{2}{|c|}{ Offset, mm } & \multicolumn{2}{|c|}{ Angle, deg } & \multicolumn{2}{|c|}{ Offset, mm } \\
\hline & Mean & $\mathrm{SD}^{\mathrm{b}}$ & Mean & SD & Mean & $\mathrm{SD}$ & Mean & SD \\
\hline$x^{\mathrm{c}}$ & 0.7 & 2.1 & 1.0 & 1.3 & 0.3 & 2.5 & 0.6 & 1.2 \\
\hline$y$ & -0.1 & 0.9 & -0.9 & 1.4 & -0.3 & 1.1 & -0.5 & 1.5 \\
\hline$z$ & 1.0 & 1.8 & -1.6 & 2.4 & 0.8 & 1.4 & -1.2 & 2.0 \\
\hline $\mathrm{RMS}^{\mathrm{d}}$ & & & 3.3 & 1.4 & & & 2.8 & 1.2 \\
\hline
\end{tabular}

${ }^{\mathrm{a}}$ VOI: Volume of interest.

${ }^{\mathrm{b}} \mathrm{SD}$ : Standard deviation of the sample.

${ }^{c} x$ : Left-right. $y$ : Posterior-anterior. $z$ : inferior-superior (perpendicular to CT image plane).

${ }^{\mathrm{d}} \mathrm{RMS}$ : root-mean square displacement.

tion differences were consistent with random error in all but one case (P7). Mean-square translation differences were consistent with random error in 7 of 12 cases. The cases in excess of random error were investigated further (P3, P4, P5, $\mathrm{P} 7, \mathrm{P} 8$, and $\mathrm{P} 10$ with a RMS translation differences ranging from 3.7 to $6.2 \mathrm{~mm}$ using the default $\mathrm{VOI}$ ).

Changes in patient geometry between the MR and CT data sets were identified as the most likely cause of registration differences. However, an average systematic difference between the MI and seed match registration metrics of 1 to $2 \mathrm{~mm}$ in the inferior-superior direction could not be ruled out.

The influences of the VOI and the control point sections on the MI registration process were investigated. To study possible negative influences of the tissues surrounding the prostate, various cropping schemes were employed. Starting with the default cropping scheme, additional cropping was used to remove, one at a time, the bladder, the bone and the

TABLE IV. Displacement of mutual information compared to seed registration as a function of volume of interest.

\begin{tabular}{|c|c|c|c|c|c|c|c|c|}
\hline \multirow[b]{2}{*}{ Patient } & \multicolumn{7}{|c|}{ Displacement, mm } & \multirow[b]{2}{*}{ Comment } \\
\hline & VOI & Default & Bladder & Bone & Rectum & Bone-SS ${ }^{\mathrm{a}}$ & Optimum $^{\text {b }}$ & \\
\hline \multirow[t]{4}{*}{ P3 } & $R_{x}$ & 1.6 & 2.7 & 1.2 & 2.9 & 1.3 & -0.7 & Bladder \\
\hline & $R_{y}$ & -1.1 & 2.4 & 0.0 & 2.9 & -0.7 & 2.3 & Reduction \\
\hline & $R_{z}$ & -4.0 & -6.1 & -5.8 & -6.4 & -4.3 & -3.3 & \\
\hline & $|R|$ & 4.4 & 7.1 & 6.0 & 7.6 & 4.6 & 4.1 & \\
\hline \multirow[t]{4}{*}{ P4 } & $R_{x}$ & 2.4 & 1.1 & 3.0 & 2.4 & 3.5 & 0.5 & Rotation, \\
\hline & $R_{y}$ & 0.3 & 0.5 & 4.0 & 0.5 & 0.9 & -0.1 & Skew \\
\hline & $R_{z}$ & -4.0 & 2.2 & -1.6 & 1.9 & -2.2 & -4.4 & \\
\hline & $|R|$ & 4.6 & 2.5 & 5.2 & 3.0 & 4.2 & 4.4 & \\
\hline \multirow[t]{4}{*}{ P5 } & $R_{x}$ & 1.9 & 1.9 & 1.3 & 1.9 & 0.7 & 1.9 & Bladder \\
\hline & $R_{y}$ & -2.4 & 2.5 & 0.3 & 2.0 & 0.2 & -0.1 & Reduction, \\
\hline & $R_{z}$ & -2.7 & -4.2 & -2.4 & -3.7 & -2.2 & -2.9 & Rectum \\
\hline & $|R|$ & 4.1 & 5.3 & 2.8 & 4.6 & 2.4 & 3.5 & Expansion \\
\hline \multirow[t]{4}{*}{ P7 } & $R_{x}$ & 1.6 & 0.3 & 0.4 & 0.5 & 1.0 & 0.5 & Rectum \\
\hline & $R_{y}$ & -2.2 & 0.4 & .4 & 0.2 & 1.4 & -0.4 & Expansion \\
\hline & $R_{z}$ & -2.6 & -2.0 & -1.4 & -1.1 & -1.7 & -1.0 & \\
\hline & $|R|$ & 3.7 & 2.0 & 1.5 & 1.3 & 2.4 & 1.2 & \\
\hline \multirow[t]{4}{*}{ P8 } & $R_{x}$ & 0.8 & 0.7 & 0.3 & 0.8 & 1.1 & 0.7 & Bladder \\
\hline & $R_{y}$ & 0.1 & 5.7 & 1.9 & 5.8 & 1.6 & -1.0 & Expansion \\
\hline & $R_{z}$ & -3.6 & -8.4 & -3.1 & -8.9 & -0.4 & -3.3 & \\
\hline & $|R|$ & 3.7 & 10.2 & 3.7 & 10.7 & 2.0 & 3.6 & \\
\hline \multirow[t]{4}{*}{ P10 } & $R_{x}$ & 1.0 & 1.2 & 1.7 & 1.4 & 1.1 & 1.2 & Bladder \\
\hline & $R_{y}$ & 1.5 & 1.8 & 0.5 & 1.8 & 0.4 & 1.0 & Reduction, \\
\hline & $R_{z}$ & -5.1 & -4.3 & -0.9 & -4.3 & -2.1 & -2.1 & Rectum \\
\hline & $|R|$ & 5.4 & 4.8 & 2.0 & 4.9 & 2.4 & 2.6 & Expansion \\
\hline
\end{tabular}

${ }^{\mathrm{a} B o n e}$ VOI with a seed match start.

${ }^{\mathrm{b}}$ Optimum VOI used, see Table V. 
rectum from the VOI. The results of the cropping study are given in Table IV (columns 3-6). Initially, three cropping strategies were attempted: Cropping out the bladder, cropping out the remainder of the bone; and cropping out the rectum.

The default cropping rules were applied independently as a test of the initial registrations. The results were consistent in all cases. In four of six cases (P5, P7, P8, P10), cropping out the remaining bone was a successful strategy. Case P8 showed a bimodal preference.

To test the dependence on the MI registration starting position, the MI process was rerun using the bone crop option with initial match points chosen from the seed images. This started the MI process at the approximate registration position preferred by the seed match registration. While the results (Bone-SS, column 7) were mostly closer to the seedmatch results, the MI registration found a minimum consistent (i.e., within random error) of the bone crop results in all cases. The remaining observed differences represented a preference of the registration metric and not an arbitrary result of the choice of the initial registration parameters. Results for case P3 remained inconsistent with estimated random error for the comparison, although the results for additional cropping were mutually consistent. Case P4 showed a preference for Bladder or Rectum cropping.

A visual comparison of image data sets was used to classify anatomical changes that had the potential of affecting data set registration. The most common causes were either bladder change or rectum change, or both (Table IV, last column). Experience in identifying changes and minimizing their effects resulted in the optimum crop guidance rules presented in Table V. The experience gained and lessons learned with the MI registration technique generally improved the approach. The above six cases were reregistered using the improved approach, with the results listed in column 7 of Table IV and in Fig. 4. In all cases the RMS displacement was less than $5 \mathrm{~mm}$.

Average differences in registration using the optimal copping rules for the twelve cases reduced the offset in the $x$ and $y$ directions to approximately $\frac{1}{2} \mathrm{~mm}$, in the $z$ direction to $-1.2 \mathrm{~mm}$ (Table III under optimum VOI). The average RMS offset for MI to seed match registration was less than $3 \mathrm{~mm}$. These improvements in offset were at the expense of an increase in the difference of angle standard deviation for the $x$ and $y$ axes. The results for the 6 cases that used default VOI's were not altered.

The MI technique may not be adequate for all cases. Optimum values for two of twelve cases showed a RMS displacement of greater than $4 \mathrm{~mm}(\mathrm{P} 3, \mathrm{P} 4)$. While this difference may not indicate an excessive systematic error in the

TABLE V. Rules for optimum volume of interest when using MI.

\begin{tabular}{ll}
\hline \hline \multicolumn{1}{c}{ Default VOI } & all bone except for $\sim 1 / 2$ of pubic bone \\
Prostate shifted relative to bone & $\begin{array}{l}\text { crop all bone and reregister } \\
\text { rectum change } \\
\text { crop most or all of rectum and reregister } \\
\text { clader change }\end{array}$ \\
\hline \hline
\end{tabular}

MI or the seed match registration procedures, it is indicative of a potential for excessive uncertainty. Case P4 experienced some skew between data sets, indicating the need for rescanning. Cases for which the bony anatomy differs by more than approximately $5 \mathrm{~mm}$ are suspect and should be reviewed for possible rescan or seed match registration. A review of a recent series of cases revealed 3 of 20 cases (15\%) requiring further attention.

Our procedure for use of MI registration is: (1) To apply the VOI cropping rules presented in Table V; (2) to identify cases with larger pubic and coccyx bone misregistrations $(>5 \mathrm{~mm})$; (3) and to evaluate these cases for possible alternative registration or rescanning.

\section{DISCUSSION}

Ideally, the post dosimetry process should be performed using a single image data set to minimize error in dose estimation to target and critical normal structures. A registration process adds an unavoidable amount of error into the relationship between the seeds and the target and normal structures volumes. One can have an excellent seed distribution (CT) with limited knowledge of the target and normal structures $(>5 \mathrm{~mm}$ error or cannot discern at all), or a limited knowledge of the seed distribution with an excellent knowledge of the target (MR). While the community is very familiar with the former, the latter was investigated by Dubois and co-workers. ${ }^{5,12}$ The error in the dose estimates due to incomplete knowledge of all of the source positions was estimated with initial optimism that it may be an acceptable replacement for routine CT-based post-implant dosimetry to determine target coverage. However, this result was later tempered by the reality of processing dosimetry on a large number of patients and the desire to improve dose calculation accuracy by removing the limitation of the source localization error. ${ }^{12}$ Particularly problematic was the poor source localization outside of the prostate gland or in highly vascularized regions, potentially introducing substantial error in the dose estimation for nearby critical structures.

The registration of sagittal and coronal data sets to the axial data set is quick and accurate since it uses all the information available. This can be accomplished because the scans are obtained during the same scanning session using the same patient setup. It is convenient to move the regis-

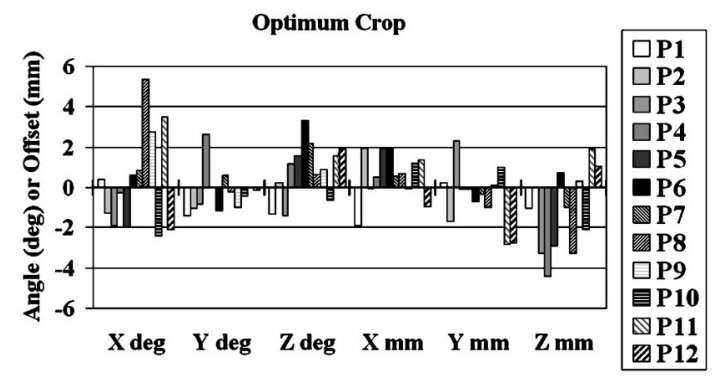

Fig. 4. Mutual information vs seed match registration offsets for optimal crop. Shown are rotation and translation offsets for twelve cases in the left-right $(x)$, posterior-anterior $(y)$, and inferior-superior $(z)$ directions. 
tered MR scans as a group to the CT scan coordinate system using the results of the axial MR to axial CT registration. Since axial scans are required for CT data sets, the axial image set is chosen as the primary dataset for the MR scans (chosen to have the smallest slice thickness) and therefore, provides the greatest amount of information common with the CT dataset. The registration of the MR data sets is best accomplished with the MI technique.

The more difficult, and therefore limiting, step in the registration process is the axial MR to axial CT registration. It is important that this step be validated carefully. There are limitations to fixing the patient in the same position for the MR and CT scan sessions. Attempts at a common setup are somewhat affected by the presence of the body coil during the MR scans. However, the more significant effect may be the time lapse between scans and the need for patient repositioning.

The MI registration technique can yield less than optimum results if (1) there is significant prostate movement between data sets $(>5 \mathrm{~mm})$ and (2) careful cropping is not used. More important is the relative state of the internal organs between scans. Even though the time between scans is minimized (40 min to $2 \mathrm{~h}$ ), we observed potentially significant changes primarily due to bladder or rectum changes. Significant differences due to anatomic distortions resulting from set-up variations are also possible. More invasive procedures, such as catheters, enemas, etc. have been considered. The current recommendation is for patient voiding before each scan. However, if on initial survey the prostate has shifted an excessive amount, the registration should be performed by seed match, or the patient rescanned. For some cases, even the seed-match procedure may not produce reliable dose estimates because of potential relative distortion of the seed distribution between scans.

A large shift in anatomy implies a differential in the relationship between the seeds and critical structures. The relationship between prostate gland and other critical structures are valid for both scans. The concern is the potential for a systematic shift in the seed distribution relative to the MR identified critical structures. A systematic error of 2 or $4 \mathrm{~mm}$ implies (at most) a dose error near the $50 \%$ gradient point of approximately $20 \%$ or $40 \%$, respectively, for I-125 implants. A mean registration error yields a probable error of much less than the maximum possible error estimate because the displacement is not always (and not likely to be) in the direction to produce the worst-case error for a particular structure.

The registration criteria used for $\mathrm{MI}$ is different from that used for seed-match registration. MI depends on the presence of similar tissue volumes, particularly muscle versus fat, in the immediate vicinity of the prostate gland. Seed matching depends on the identification of seed locations mainly in the prostate. Seed matching is more prone to rotation error but in principle produces a better estimate of the center-of-mass of the seed distribution. MI registration is more sensitive to the average position of the surrounding tissues. There is little evidence of a systematic error common to both techniques, beyond using common data sets. The net expected maximum error in the registration comparison is $\sim 3 \mathrm{~mm}$ because of the expected noncorrelated error in each of the techniques and propagation of error. A difference greater than $3 \mathrm{~mm}$ implies the presence of systematic error. An important part of the registration process is being able to predict when the MI registration will imply a potential error in the location of the seed distribution.

Seed match registration used here was performed thoroughly and double checked in an effort to define an optimal registration standard. However, due to the systematic process of identifying seeds and defining appropriate matched pairs and the potential for seed distribution distortions between data sets, a minimum systematic error remained. Seed match registration error approaches a minimum for a relatively small number of appropriately chosen seed positions.

The advantage of MI registration over seed match registration is work efficiency (i.e., speed). The step requiring the greatest experience and care is the seed pair identification. Auto seed identification on MR and auto MR-CT seed pair identification is not error-free and requires a verification step. While it is possible to optimize the seed match procedure, MI registration retains the advantage unless there is significant anatomic distortion. When the evidence of anatomic distortion are sufficiently strong, seed match registration is preferred. Current procedures specify approximately 10 seeds be chosen for match, evenly distributed over the implant volume. The seed-match procedure remains time consuming relative to the MI procedure.

Accepting the added uncertainty of registration allows one to limit and somewhat control the total level of uncertainty in the process. Given the nature of the information contained in the data sets (e.g., $1 \times 1 \times 2 \mathrm{~mm}^{3}$ or larger voxel sizes), a $\sim 2 \mathrm{~mm}$ registration error is expected for the seed match registration, ${ }^{13}$ bone to bone registration ${ }^{14}$ or MI registration (present work). The impact of this registration uncertainty must be less than the advantage gained by having access to the second data set. At present, the advantage of having the MR data set is the ability to estimate dose to critical normal structures as part of the continuing effort to place dose where it is needed while avoiding dose where it is not needed and results in unnecessary discomfort or dysfunction. Two to three millimeters of uncertainty in the position of the target and normal structures is a small price compared to not having specific information on their location, or worse setting target boundaries larger than necessary.

\section{CONCLUSION}

The use of mutual information for the registration of MR and CT data sets post implant of the prostate allows efficient use of a large amount of information and can be used routinely with appropriate precautions for the identification of cases compromised by excessive shifting of internal structures (i.e., prostate, bladder, rectum) between scans. Care should be used to limit anatomical differences between scans regardless of the registration technique. Minimum axial MR to axial CT registration error is on the order of $2 \mathrm{~mm}$ and is limited by the resolution of the data sets. 
${ }^{a)}$ Author to whom correspondence should be addressed. Department of Radiation Oncology, University of Michigan, UH-B2C490, 1500 E. Medical Center Dr., Ann Arbor, MI 48109. Telephone: 734-936-4309; Fax: 734-936-2261. Electronic mail: roberpl@umich.edu

${ }^{1}$ S. Nag, W. Bice, K. deWyngaert, B. Prestidge, R. Stock, and Y. Yu, "The American Brachytherapy Society recommendations for permanent prostate brachytherapy postimplant dosimetric analysis," Int. J. Radiat. Oncol., Biol., Phys. 46, 221-230 (2000).

${ }^{2}$ M. Roach, P. Faillace-Akazawa, C. Malfatti, J. Holland, and H. Hricak, "Prostate volumes defined by magnetic resonance imaging and computerized tomographic scans for three-dimensional conformal radiotherapy," Int. J. Radiat. Oncol., Biol., Phys. 35, 1011-1018 (1996).

${ }^{3}$ P. W. McLaughlin, V. Narayana, D. G. Drake, B. M. Miller, L. Marsh, J. Chan, R. Gonda, Jr., R. J. Winfield, and P. L. Roberson, "Comparison of MRI pulse sequences in defining prostate volume after permanent implantation," Int. J. Radiat. Oncol., Biol., Phys. 54, 703-711 (2002).

${ }^{4}$ M. Milosevic, S. Voruganti, R. Blend, H. Alasti, P. Warde, M. Mclean, P. Catton, C. Catton, and M. Gospodarowicz, "Magnetic resonance imaging (MRI) for localization of the prostate apex: comparison to computed tomography (CT) and urethrography," Radiother. Oncol. 47, 277-284 (1998).

${ }^{5}$ D. F. Dubois, B. R. Prestidge, L. A. Hotchkiss, W. S. Bice, Jr., and J. J. Prete, "Source localization following permanent transperineal prostate interstitial brachytherapy using magnetic resonance imaging," Int. J. Radiat. Oncol., Biol., Phys. 39, 1037-41 (1997).

${ }^{6}$ D. F. Dubois, B. R. Prestidge, L. A. Hotchkiss, J. J. Prete, and W. S. Bice, Jr., "Intraobserver and interobserver variability of MR imaging and CTderived prostate volumes after transperineal interstitial permanent pros- tate brachytherapy," Radiology 207, 785-789 (1998).

${ }^{7}$ R. J. Amdur, D. Gladstone, K. A. Leopold, and R. D. Harris, "Prostate seed implant quality assessment using MR and CT image fusion," Int. J. Radiat. Oncol., Biol., Phys. 43, 67-72 (1999).

${ }^{8}$ W. M. Wells, P. Viola, H. Atsume, S. Nakajima, and R. Kikinis, "Multimedia volume registration by maximization of mutual information," Med. Image Anal. 1, 35-51 (1996).

${ }^{9}$ F. Maes, A. Collignon, D. Vandermeulen, G. Marchal, and P. Suetens, "Multimodality image registration by maximization of mutual information," IEEE Trans. Med. Imaging 16, 187-198 (1997).

${ }^{10}$ D. L. McShan, R. K. Ten Haken, and B. A. Fraass, "3D Treatment planning: IV integrated brachytherapy planning," in The Use of Computers in Radiation Therapy, edited by I. A. D. Bruinvis et al. (Elsevier Science Publishers B.V., North-Holland, 1987).

${ }^{11}$ P. Bourel, D. Gibon, E. Coste, V. Daanen, and J. Rousseau, "Automatic quality assessment protocol for MRI equipment," Med. Phys. 26, 26932700 (1999)

${ }^{12}$ J. J. Prete, B. R. Prestidge, W. S. Bice, D. F. Dubois, and L. A. Hotchkiss, "Comparison of MRI- and CT-based post-implant dosimetric analysis of transperineal interstitial permanent prostate brachytherapy," Radiat. Oncol. Invest. 6, 90-96 (1998).

${ }^{13}$ D. F. Dubois, W. S. Bice, and B. R. Prestidge, "CT and MRI derived source localization error in a custom prostate phantom using automated image coregistration,” Med. Phys. 28, 2280-2284 (2001).

${ }^{14}$ R. C. Zellars, P. L. Roberson, M. Strawderman, D. Zhang, H. Sandler, R. K. Ten Haken, D. Osher, and P. W. McLaughlin, "Prostate position late in the course of external beam therapy: Patterns and predictors," Int. J. Radiat. Oncol., Biol., Phys. 47, 655-660 (2000). 